\title{
Sobre dimensiones de Modelos de Gel'fand
}

\author{
Dr. José O. Araujo \\ NuCOMPA-Facultad de Ciencias Exactas, UNICEN \\ Paraje Arroyo Seco s/n, 7000, Tandil, Argentina \\ Araujo, J. O. araujo@exa.unicen.edu.ar \\ Paz, K. A. kapaz@exa.unicen.edu.ar
}

(recibido/received: 14-Oct-2008; aceptado/accepted: 27-Marzo-2009)

\section{RESUMEN}

En este artículo se presenta una expresión para la dimensión de un modelo de Gel'fand del grupo simétrico generalizado $\mathfrak{S}_{n}^{m}$ o grupo de reflexiones unitarias de tipo $G(m, 1, n)$. De la misma se concluye que esta dimensión coincide con el número de elementos que se representan como productos de reflexiones cuyas raíces son ortogonales dos a dos.

\begin{abstract}
In this article we present the term for the expression of the Gel'fand model belonging to the generalized symmetrical group $\mathfrak{S}_{n}^{m}$ or group of unitary consideration type $G(m, 1, n)$.From this we conclude that this aspect coincides with the number of elements which are represented as products of reflection the roots of which are orthogonal two to two.
\end{abstract}

\section{Introducción.}

Un modelo de Gel'fand para un grupo finito $G$, es una representación ordinaria de $G$ cuyo carácter es la suma de todos los caracteres irreducibles de $G$.

Notaremos con $\gamma(G)$ a la dimensión de un modelo de Gel'fand para el grupo finito $G$. Un resultado particularmente interesante sobre $\gamma(G)$, se da en la situación en que todas las representaciones irreducibles de $G$ pueden ser realizadas sobre los números reales. En tal caso, como consecuencia del indicador de Fröbenius-Schür, ver [James] o [Curtis], se obtiene que $\gamma(G)$ es igual al número de involuciones en $G$, es decir:

$$
\gamma(G)=\left|\left\{\sigma \in G: \sigma^{2}=e\right\}\right|
$$

aquí las barras indican el cardinal del conjunto y $e$ es el elemento neutro de $G$.

En el caso de los grupos de reflexiones euclidianas, ver [Springer], todas las representaciones de un grupo de Weyl pueden ser realizadas sobre los números racionales, mientras que para los grupos de Coxeter $\mathrm{H}_{3}$ y $\mathrm{H}_{4}$ las representaciones se realizan sobre los números reales. Naturalmente que esta información es de suma utilidad a la hora de estudiar modelos de Gel'fand para estos grupos.

En el caso del grupo simétrico generalizado o grupo de reflexiones unitarias $G(m, 1, n)$, las representaciones pueden realizarse sobre extensiones ciclotómicas, ver [Can, H.]

Para la teoría de grupos de reflexiones en general puede consultarse [Cohen],[Hum],[Kane] y [ShephTodd]. 


\section{O. Araujo}

\section{Construcciones de modelos de Gel'fand.}

Este concepto de modelo de Gel'fand aparece hacia 1981, justamente a partir del trabajo de Bernstein, Gel'fand y Gel'fand en [BGG], donde presentan modelos para grupos de Lie compactos. A partir de entonces hay diversas construcciones de modelos de Gel'fand realizadas por distintos autores.

En [Klyachko], Klyachko presenta un modelo para $G l_{n}\left(F_{q}\right)$ presentado con suma de caracteres inducidos. En [PanSoto], Pantoja y Soto-Andrade lo hacen para un grupo de Movimientos rígidos, en [IngSaxl], Inglis y Saxl para el grupo simétrico, en [Bad], Baddeley estudia modelos por involuciones, en [HZ], Howlett y Zworestine retoman el modelo de Klyachko desde un enfoque más actual.

Kodiyalam y Verma en [KodiyaVer], presenta una construcción particularmente simple de modelo para el grupo simétrico desarrollado sobre la potencia exterior $K^{n} \wedge K^{n}$. Finalmente, Adin, Postnikov y Roichman, en [APR1] y [APR], lo hacen para el grupo simétrico y su álgebra de Iwahori-Hecke y para el grupo simétrico generalizado.

En [AA] se introduce un espacio $\mathcal{N}$, en anillo de funciones polinomiales $\mathcal{A}=K\left[x_{1}, \ldots, x_{n}\right]$, asociado con un subgrupo finito $G$ de $G l_{n}(K)$. El espacio $\mathcal{N}$ es un subespacio del espacio de polinomios $G$-armónicos, es decir del espacio:

$$
\left\{P \in \mathcal{A}: \partial_{Q}(P)=0, \forall Q \in \mathcal{A}_{+}^{G}\right\}
$$

donde $\mathcal{A}_{+}^{G}$ son los polinomios $G$-invariantes con término constante igual a cero y:

$$
\partial_{Q}=Q\left(\partial_{x_{1}}, \ldots, \partial_{x_{n}}\right)
$$

Por otra parte:

$$
\mathcal{N}=\left\{P \in \mathcal{A}: \mathcal{D}(P)=0, \forall D \in \mathcal{W}_{+}^{G}\right\}
$$

donde $\mathcal{W}_{+}^{G}$ son los operadores diferenciales $G$-invariantes en el álgebra de Weyl de la forma:

$$
D=\sum_{\alpha, \beta} x^{\alpha} \partial^{\beta} \text { con }|\alpha|<|\beta|
$$

siendo:

$$
\begin{array}{cc}
x^{\alpha}=x_{1}^{\alpha_{1}} \cdots x_{n}^{\alpha_{n}} & \partial^{\beta}=\partial_{x_{1}}^{\beta_{1}} \cdots \partial_{x_{n}}^{\beta_{n}} \\
|\alpha|=\alpha_{1}+\cdots+\alpha_{n} & |\beta|=\beta_{1}+\cdots+\beta_{n}
\end{array}
$$

Cuando $K$ es algebraicamente cerrado, la representación natural de $G$ sobre $\mathcal{N}$ contiene una copia de cada representación irreducible de $G$. Particularmente interesante es el caso en que $G$ es un grupo de reflexiones, es conocido que si $G$ es un grupo de reflexiones, ver [Kane], la representación de $G$ sobre los polinomios $G$-armónicos es equivalente a la representación regular. Para los grupos diedrales, los grupos clásicos de tipo $A_{n}, B_{n}, D_{2 n+1}$ y para el grupo $G(n, 1, m)$ (o grupo simétrico generalizado), $\mathcal{N}$ es un modelo de Gel'fand, ver [AA1], [A], [AB] y [AB1].

En el tratamiento de los grupos de tipo $B_{n}$ y $D_{n}$, se hace uso del hecho que las representaciones de un grupo de Weyl pueden ser realizadas sobre los números racionales, ver [Springer], y en consecuencia, 


\section{O. Araujo}

del conocimiento previo de la dimensión de un modelo de Gel'fand como el número de involuciones en el grupo.

Para un grupo diedral con $2 n$ elementos, se mantiene que $\mathcal{N}$ un modelo de Gel'fand tiene por dimensión al número de involuciones en el grupo, es decir $n+2$ o $n+1$ según $n$ sea par o impar. Es este caso se puede ver que $\mathcal{N}$ en $\mathbb{C}[x, y]$ el espacio de polinomios armónicos que son anulados por los operadores:

$$
x^{k} \partial_{y}^{m-k}+y^{k} \partial_{x}^{m-k} \quad 1 \leq k \leq n
$$

\section{Producto semidirectos.}

El estudio de las representaciones irreducibles en productos semidirectos de grupos, presenta un caso destacable por su simplicidad. Este es el caso de un grupo finito $G$ que se presenta como producto semidirecto de un subgrupo normal abeliano $D$ y un subgrupo $H$, es decir:

$$
G=D \times{ }_{s} H
$$

La construcción de las representaciones irreducibles en este caso puede ser obtenida como se describe a continuación, ver por ejemplo [Serre] o [Curtis].

Con cada carácter lineal $\chi$ de $D$ consideramos el grupo de inercia de $\chi$ en $H$ dado por:

$$
I_{\chi}=\left\{\eta \in H: \chi^{\eta}=\chi\right\}
$$

donde:

$$
\chi^{\eta}(d)=\chi\left(\eta d \eta^{-1}\right) \quad d \in D
$$

Ahora, con cada representación irreducible $\mu$ de $I_{\chi}$, definimos una representación $\chi \mu$ de $D \times_{s} I_{\chi}$ definida por:

$$
\chi \mu(d, \sigma)=\chi(d) \mu(\sigma) \quad d \in D, \sigma \in I_{\chi}
$$

Dado que $D$ es un subgrupo normal y $\chi$ es un carácter lineal, $\chi \mu$ queda bien definida sobre el espacio de representación de $\mu$. Notamos con $\rho_{\chi \mu}$ a la representación de $G$ inducida por $\chi \mu$.

Si $\mathcal{R}$ una familia de representantes de las $G$-órbitas en el espacio de caracteres lineales de $D$, entonces las representaciones irreducibles de $G$ pueden ser parametrizadas como:

$$
\widehat{G}=\left\{\rho_{\chi \mu}: \chi \in \mathcal{R}, \mu \in \widehat{I_{\chi}}\right\}
$$

donde el sombrero indica, salvo equivalencias, el conjunto de todas de representaciones irreducibles del grupo considerado.

De lo anterior resulta: 


$$
\begin{aligned}
\gamma(G) & =\sum_{(\chi, \mu) \in \mathcal{R} \times \widehat{I_{\chi}}}\left[H: I_{\chi}\right] \mu(e) \\
& =\sum_{\chi \in \mathcal{R}}\left[H: I_{\chi}\right]\left(\sum_{\mu \in \widehat{I_{\chi}}} \mu(e)\right) \\
& =\sum_{\chi \in \mathcal{R}}\left[H: I_{\chi}\right] \gamma\left(I_{\chi}\right) .
\end{aligned}
$$

\section{El grupo simétrico $\mathfrak{S}_{n}$.}

En el caso del grupo simétrico, las involuciones se expresan en forma única como producto de transposiciones dos a dos disjuntas, en consecuencia éstas pueden ser agrupadas según el número de transposiciones en que se descomponen.

Para un subgrupo $\mathfrak{H}$ de $\mathfrak{S}_{n}$, notaremos con $\mathcal{I}_{k}(\mathfrak{H})$, con $0 \leq k \leq\left[\frac{n}{2}\right]$, al conjunto de las involuciones en $\mathfrak{H}$ que se descomponen como producto de $k$ involuciones dos a dos disjuntas. También usaremos $\gamma_{k}(\mathfrak{H})$ para indicar el cardinal de $\mathcal{I}_{k}(\mathfrak{H})$.

Sin mayor dificultad puede establecerse que:

$$
\gamma_{k}\left(\mathfrak{S}_{n}\right)=\frac{n !}{2^{k} k !(n-2 k) !}
$$

Con cada una partición $\lambda=\left(\lambda_{1}, \cdots, \lambda_{k}\right)$ de $n$, asociamos el subgrupo parabólico $\mathfrak{S}_{\lambda}$ de $\mathfrak{S}_{n}$ dado por:

$$
\mathfrak{S}_{\lambda}=\mathfrak{S}_{\lambda_{1}} \times \cdots \times \mathfrak{S}_{\lambda_{k}}
$$

donde cada $\mathfrak{S}_{\lambda_{j}}$ está dado por:

$$
\mathfrak{S}_{\lambda_{j}}=\left\{\sigma \in \mathfrak{S}_{n}: \sigma(i)=i \operatorname{si} \lambda_{1}+\cdots+\lambda_{j-1}<i \leq \lambda_{1}+\cdots+\lambda_{j}\right\} .
$$

En virtud de la caracterización de las representaciones irreducibles de un producto cartesiano, se tiene:

Proposición i) Las representaciones irreducibles de $S_{\lambda}$ pueden ser realizadas sobre los números reales.

ii) $\gamma\left(\mathfrak{S}_{\lambda}\right)$ es el número de involuciones en $\mathfrak{S}_{\lambda}$ y se tiene:

$$
\gamma\left(\mathfrak{S}_{\lambda}\right)=\sum_{k=0}^{\left[\frac{n}{2}\right]} \gamma_{k}\left(\mathfrak{S}_{\lambda}\right) \text {. }
$$

\section{El grupo simétrico generalizado $\mathfrak{S}_{n}^{m}$.}

Como ya hemos mencionado, $\gamma(G)$ es el número de involuciones en $G$ en el caso de los grupos de reflexiones euclidianas. En virtud de los resultados de Carter en [Carter], en este caso, toda involución puede ser expresada como producto de reflexiones cuyas raíces forman un sistema ortogonal, de modo que resultan equivalentes ser involución y expresarse como producto de reflexiones asociadas a un sistema ortogonal de raíces. 


\section{O. Araujo}

En un grupo de reflexiones unitarias $G$, llamaremos pseudo-involuciones a los elementos en $G$ que puedan ser expresados como producto de reflexiones asociadas a un sistema ortogonal de raíces. El resultado que presentamos en este breve artículo es el siguiente:

Teorema 5.1. i) $\gamma\left(\mathfrak{S}_{n}^{m}\right)$ coincide con el número de pseudo-involuciones en $\mathfrak{S}_{n}^{m}$. ii)

$$
\gamma\left(\mathfrak{S}_{n}^{m}\right)=\sum_{k=0}^{\left[\frac{n}{2}\right]} m^{n-k} \frac{n !}{2^{k} k !(n-2 k) !} .
$$

Demostración: Presentamos el grupo simétrico generalizado como su representación geométrica dada por las matrices monomiales en $\mathbb{C}^{n \times n}$, cuyos coeficientes no nulos son raíces $m$-ésimas de la unidad. En esta situación se tiene:

$$
\mathfrak{S}_{n}^{m}=\mathfrak{D} \times{ }_{s} \mathfrak{S}_{n}
$$

donde $\mathfrak{D}$ es el grupo de matrices diagonales raíces $m$-ésimas de la unidad en la diagonal principal y $\mathfrak{S}_{n}$ es el grupo simétrico identificado con las matrices permutacionales.

Resulta claro que el grupo de inercia de un carácter lineal de $\mathfrak{D}$ en $\mathfrak{S}_{n}$ es conjugado con un subgrupo parabólico $\mathfrak{S}_{\lambda}$ para alguna partición $\lambda$ de $n$. Por otra parte, cada subgrupo parabólico de $\mathfrak{S}_{n}$ es el grupo de inercia en $\mathfrak{S}_{n}$ de algún carácter lineal de $\mathfrak{D}$.

Notamos con $\mathcal{F}_{n}^{m}$ el conjunto de funciones de $\{1,2, \ldots, n\}$ a valores en $\{1,2, \ldots, m\}$. Cada elemento $\alpha \in \mathcal{F}_{n}^{m}$ induce una partición de $n$ dada por:

$$
\lambda_{i}^{\alpha}=\left|\alpha^{-1}(i)\right| \quad 0 \leq i \leq m
$$

Notemos que cada carácter lineal $\chi$ de $\mathfrak{D}$ puede identificarse naturalmente con una función en $\mathcal{F}_{n}^{m}$ . Es este caso, dos caracteres lineales $\chi$ y $\phi$ de $\mathfrak{D}$ son equivalentes si existe $\pi \in \mathfrak{S}_{n}$ tal que:

$$
\phi=\chi^{\pi}
$$

pero, pensados como funciones, la identidad precedente se traduce en:

$$
\alpha_{\phi}=\alpha_{\chi} \circ \pi^{-1}
$$

de modo que el número de caracteres en la $\mathfrak{S}_{n}$-órbita de $\chi$ está dado por:

$$
\frac{n !}{\lambda^{x} !}=\left[\mathfrak{S}: \mathfrak{S}_{\lambda^{x}}\right]
$$

De este modo, la expresión:

$$
\gamma\left(\mathfrak{S}_{n}^{m}\right)=\sum_{\chi \in \mathcal{R}}\left[\mathfrak{S}: \mathfrak{S}_{\lambda^{x}}\right] \gamma\left(\mathfrak{S}_{\lambda^{x}}\right)
$$

puede ser reescrita como: 


$$
\begin{aligned}
\gamma\left(\mathfrak{S}_{n}^{m}\right) & =\sum_{\alpha \in \mathcal{F}_{n}^{m}} \gamma\left(\mathfrak{S}_{\lambda^{\alpha}}\right) \\
& =\sum_{k=0}^{\left[\frac{n}{2}\right]} \sum_{\alpha \in \mathcal{F}_{n}^{m}} \gamma_{k}\left(\mathfrak{S}_{\lambda^{\alpha}}\right) .
\end{aligned}
$$

En la suma:

$$
\sum_{\alpha \in \mathcal{F}_{n}^{m}} \gamma_{k}\left(\mathfrak{S}_{\lambda^{\alpha}}\right)
$$

cada involución $l \in \mathcal{I}_{k}\left(\mathfrak{S}_{n}\right)$ aporta tantas unidades como la cantidad de subgrupos parabólicos que la contienen. Pensando de otra manera, podemos ver el número de $\alpha \in \mathcal{F}_{n}^{m}$ tales que $\imath \in \mathfrak{S}_{\lambda_{\alpha}}$. Pero esto es:

$$
m^{k} \times m^{n-2 k}=m^{n-k}
$$

dado que $\alpha$ debe tomar valores constantes sobre ambos índices de las transposición disjuntas que interviene en la descomposición de $\boldsymbol{l}$.

Se concluye que:

$$
\gamma\left(\mathfrak{S}_{n}^{m}\right)=\sum_{k=0}^{\left[\frac{n}{2}\right]} m^{n-k} \times \gamma_{k}\left(\mathfrak{S}_{n}\right)
$$

o también , en virtud de (1):

$$
\gamma\left(\mathfrak{S}_{n}^{m}\right)=\sum_{k=0}^{\left[\frac{n}{2}\right]} m^{n-k} \frac{n !}{2^{k} k !(n-2 k) !}
$$

Por otra parte, resulta simple ver que tanto el número de matrices simétricas, el número de pseudoinvoluciones proyectivas coincide con:

$$
\sum_{k=0}^{\left[\frac{n}{2}\right]} m^{n-k} \times \gamma_{k}\left(\mathfrak{S}_{n}\right)
$$

\section{REFERENCIAS}

[1]. Adin, R. M., Postnikov, A., Roichman, Y., A Gelfand model for Wreath Products, arXiv:math.RT/08022824 v1, 2008.

[2]. Adin, R. M., Postnikov, A., Roichman, Y, Comobinatorial Gelfand Models, arXiv:math.RT/07093962 v2, 2008.

[3]. Aguado, J. L. and Araujo, J. O., A Gelfand model for the symmetric group, Communications in Algebra, 29 (4), 1841 - 1851 (2001).

[4]. Aguado, J. L. and Araujo, J. O., Representations of Finite Groups on Polynomial Rings. Actas V Congreso de Matemática Dr. Antonio R. Monteiro, 35-40 (1999) Bahía Blanca.

[5]. Araujo, J.O., A Gelfand model for a Weyl group of type Bn, Beiträge zur Algebra und Geometrie 44, no. 2 (2003) 359-373.

[6]. Araujo, J. O. and Bigeón, J. J., A Gelfand Model for the Weyl group of type $D_{n}$ and the 


\section{O. Araujo}

branching rules $D_{n} \hookrightarrow B_{n}$. Journal in Algebra, vol. 294, (2005), 97-116.

[7]. Araujo, J. O. and Bigeón, J. J., A Gelfand Model for the Symmetric Generalized Group, por aparecer en Communications in Algebra.

[8]. Baddeley, R., Models and Involution Models for Wreath Products and certain Weyl Groups. Journal of London Mathematical Society no. 44, serie 2 (1991) 55-74.

[9]. Bernstein, I, Gelfand, I. and Gelfand, S. Models of representations of Lie groups, Selected. Math. Soviet 1 (2) (1981) 121-142.

Can, H. Representations of the generalized symmetric groups. Beitr. Algebra Geom. 37, No.2, 289-307 (1996).

[1]. Carter, R. W. Conjugacy classes in the Weyl group. Compositio Math. 25 (1972), 159.

[2]. Cohen, A. M. Finite complex reflection groups. Ann. scient. 'Ec. Norm. Sup. 9 (1976), 379-436. Erratum: ibid. 11 (1978), 613.

[3]. Curtis, C. W. \& Reiner, I. Methods of Representation theory with Applications to Finite Groups and Orders. Vol. I, John Wiley \& Sons, 1981.

[4]. Howlett, R. and Zworestine, C., On Klyachkos model for the representations of finite linear groups. China Higher Education Press (Beijing), and Springer-Verlag (Berlin, Heidelberg), (2000), 229-246.

[5]. Humphreys, J. E. Reflection groups and Coxeter groups. Cambridge Stud. Adv. Math., vol. 29, Cambridge University Press, 1990.

[6]. Inglis, N. F. J. and Saxl, J., An explicit model for the complex representations of the finite general linear groups, Archiv der Mathematik 57 (1991), 424-431.

[7]. James, G. and Liebeck, M. Representations and characters of groups. Cambridge Mathematical Textbooks. 1993.

[8]. Kane, R. Reflection groups and invariant theory. CMS Books in Mathematics 5, Springer, New York, 2001.

[9]. Klyachko, A. A., Models for the complex representations of the groups $G L(n, q)$, Math. USSR Sbornik 48 (1984), 365379.

[1]. Kodiyalam, V. and Verma, D.N., A natural representation model for symmetric groups. arXiv:math.RT/0402216 v1, 2006.

[2]. Pantoja, J. and Soto-Andrade, J., Fonctions sphériques et modèles de Gel'fand pour le groupe de mouvements rigides d'un espace paraeuclidien sur un corps local. Comptes Rendus de L'Académie des Sciences 302, (1986), 463-466.

[3]. Serre, J. P. Representaciones lineales de grupos finitos. Ediciones Omega S. A. Barcelona 1970.

[4]. Shephard, G. C.and Todd, J. A. Finite unitary reflection groups. Canad. J. Math. 6 (1954), 274304.

[5]. Springer, T., A Construction of Representations of Weyl Groups. Inventiones Mathematicae 44 (1978) 279-293. Sciences 302, (1986), 463-466.

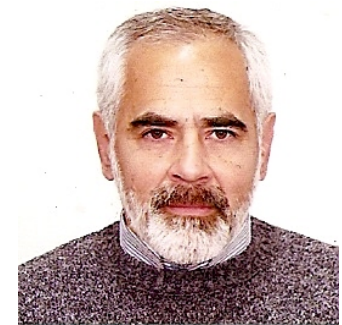

Dr. José Orlando Araujo

Profesor Titular

Departamento de Matemática

Facultad de Ciencias Exactas

Universidad Nacional del Centro de la Provincia de Buenos Aires 
O. Araujo

Vol. 21, No. 02, pp. 77-83 / Abril 2009 